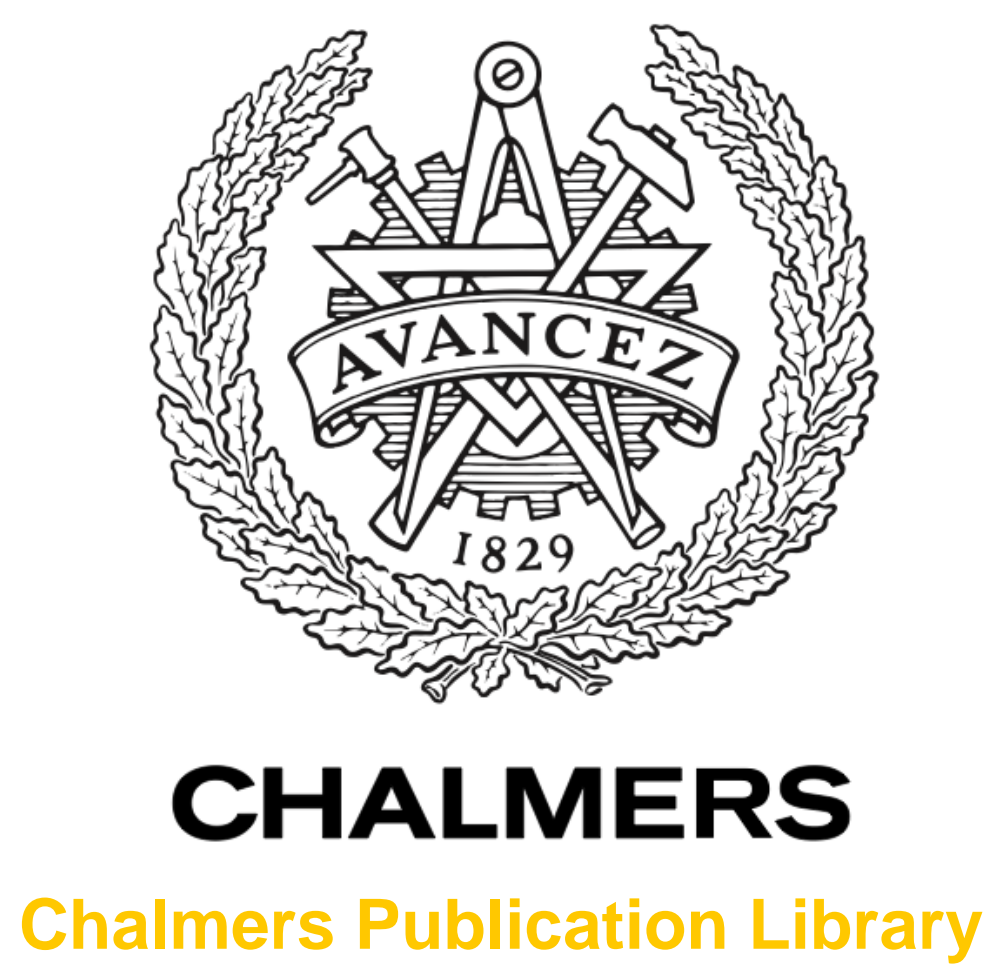

\title{
Efficient Wideband Gain Modeling for Interferometric Imaging Arrays in Radio
} Astronomy

This document has been downloaded from Chalmers Publication Library $(\mathrm{CPL})$. It is the author's version of a work that was accepted for publication in:

\section{IEEE International Symposium on Antennas and Propagation \& USNC/URSI National} Radio Science Meeting

Citation for the published paper:

Young, A. ; Davidson, D. ; Wijnholds, S. et al. (2015) "Efficient Wideband Gain Modeling for Interferometric Imaging Arrays in Radio Astronomy". 2015 IEEE International

Symposium on Antennas and Propagation \& USNC/URSI National Radio Science Meeting

http://dx.doi.org/10.1109/APS.2015.7305076

Downloaded from: http://publications.lib.chalmers.se/publication/227915

Notice: Changes introduced as a result of publishing processes such as copy-editing and formatting may not be reflected in this document. For a definitive version of this work, please refer to the published source. Please note that access to the published version might require a subscription. 


\section{Efficient Wideband Gain Modeling for Interferometric Imaging Arrays in Radio Astronomy}

\author{
A. Young, D. B. Davidson \\ University of Stellenbosch \\ Stellenbosch, South Africa
}

\author{
S. J. Wijnholds \\ ASTRON \\ Dwingeloo, The Netherlands
}

\author{
T. D. Carozzi, R. Maaskant, M. V. Ivashina \\ Chalmers University of Technology \\ Gothenburg, Sweden
}

\begin{abstract}
Current and future interferometric imaging arrays in radio astronomy may be limited by the accuracy with which various direction-dependent effects, such as the antenna gain patterns, can be corrected. Doing this in an efficient manner during the calibration and imaging process is especially difficult due to these effects often being also baseline-dependent. A newly proposed method, called A-stacking, offers a solution through the use of a parametrized model in which basis functions account for the direction-dependence and the expansion coefficients account for the baseline-dependence. This approach provides a simple trade-off between computational complexity and calculation accuracy. Herein the use of the A-stacking method to model direction-dependent effects over a very wide frequency band is investigated.
\end{abstract}

\section{INTRODUCTION}

Imaging with an interferometer array is based on the Van Cittert-Zernike theorem which relates the intensity distribution on the sky to the visibility function on a plane in the form of a two-dimensional Fourier transform [1]. By measuring cross-correlations between the different antenna pairs (called baselines) the visibility function is sampled, and an image of the sky may be obtained by applying the inverse Fourier transform. Inversion is rarely done directly - the reasons for this include general non-uniqueness of the solution and the scale of the calculations involved - and an iterative algorithm is usually applied, which involves the following steps: 1) transformation of the visibilities into an image (backward calculation); 2) analysis of the image to derive / update a model of the imaged sky; and 3) transformation of the sky model to model visibilities (forward calculation) to test the solution accuracy.

The efficiency of the Fast Fourier Transform (FFT) is often employed to expedite the forward and backward calculations [2]. One difficulty in using the FFT arises due to the effect of the antenna gain patterns on the visibility measurements. Since the direction-dependent distortion caused by these gains is generally different on each baseline - as they depend on various antenna-based factors such as manufacturing tolerances of the antennas, changing environmental conditions, and mutual coupling - the measured visibilities over the entire array are no longer related to a single distorted sky, but each baseline measures visibilities pertaining to a differently distorted sky [3].

One very recently proposed solution to this problem, called A-stacking [4], provides a separation of direction-dependence and baseline-dependence through the use of an appropriately defined linear model. Another advantage of this approach is that it yields a simple trade-off between computational complexity and calculation accuracy. In this contribution this tradeoff is considered in the forward calculation for a wideband imaging array.

\section{A-STACKING Formulation}

Using a simplistic linear model the response of an ideal interferometric array to a given sky model may be compactly written as,

$$
\mathbf{v}=\boldsymbol{\Phi} \sigma .
$$

Here $\sigma$ is an $N_{p}^{2} \times 1$ vector in which each element represents a pixel intensity (the sky brightness) in an $N_{p} \times N_{p}$ model image of the sky, $\mathbf{v}$ is a $K \times 1$ vector containing the complexvalued model visibilities measured by the array, and $\boldsymbol{\Phi}$ is a matrix that represents the Fourier transform with the element on the $k$ th row (baseline index) and $n$th column (pixel index) equal to,

$$
\phi_{k}^{(n)}=e^{-j 2 \pi\left(u_{k} l_{n}+v_{k} m_{n}\right)} .
$$

The wavelength normalized spatial frequency (visibility) space coordinates are $(u, v)$, and $(l, m)$ are the related direction cosines in the image plane. The Fourier transform in (1) may be efficiently performed via the FFT and an additional degridding operation [2].

By introducing the antenna gain patterns in the model, which are both direction-dependent and baseline-dependent, the equivalent of (1) becomes,

$$
\mathbf{v}=(\mathbf{\Phi} \odot \mathbf{B}) \boldsymbol{\sigma},
$$

where $\odot$ is the element-wise, or Hadamard product, and

$$
\mathbf{B}=\left[\begin{array}{llll}
\mathbf{b}_{1} & \mathbf{b}_{2} & \cdots & \mathbf{b}_{K}
\end{array}\right]^{\mathrm{T}} .
$$

Here each $\mathbf{b}_{k}$ is an $N_{p}^{2} \times 1$ complex-valued vector discretization over the image plane of the cross-power pattern of the antennas that comprise the $k$ th baseline. Due to the distortion B, the transformation in (3) is not in a form which allows an efficient forward calculation via the FFT; within the A-stacking framework (3) is recast in a form suitable for that purpose.

Using A-stacking a suitable basis is sought that allows each $\mathbf{b}_{k}$ to be written as [4],

$$
\mathbf{b}_{k}=\sum_{i=1}^{N_{B}} a^{(i, k)} \mathbf{f}_{i},
$$


wherein the coefficients $\left\{a^{(i, k)}\right\}$ account for the baselinedependence, and the basis functions $\left\{\mathbf{f}_{i}\right\}$ account for the direction-dependence of the gains associated with the antennas. Substituting this model into (3) gives

$$
\mathbf{v}=\sum_{i=1}^{N_{B}} \operatorname{diag}\left(\mathbf{a}_{i}\right) \boldsymbol{\Phi} \operatorname{diag}\left(\mathbf{f}_{i}\right) \boldsymbol{\sigma},
$$

where $\mathbf{a}_{i}=\left[\begin{array}{llll}a^{(i, 1)} & a^{(i, 2)} & \cdots & a^{(i, K)}\end{array}\right]^{\mathrm{T}}$. Now the model visibilities can be calculated efficiently as follows:

1) A per-basis function image domain correction is applied, $\boldsymbol{\sigma}_{i}=\operatorname{diag}\left(\mathbf{f}_{i}\right) \boldsymbol{\sigma}$.

2) Corresponding sets of visibilities are computed, $\mathbf{v}_{i}=$ $\boldsymbol{\Phi} \boldsymbol{\sigma}_{i}$. This may be done efficiently via the FFT and degridding.

3) A correction is applied to each set of visibilities, and the results for all basis functions accumulated, $\mathbf{v}=$ $\sum_{i}^{N_{B}} \operatorname{diag}\left(\mathbf{a}_{i}\right) \mathbf{v}_{i}$.

The above process is highly parallelizable, since the computation from each $\sigma_{i}$ to each $\mathbf{v}_{i}$ can be performed independently. Furthermore, the overall computational cost scales linearly with the number of basis functions required to model the direction-dependent gains over all baselines with sufficient accuracy. Hence it is advantageous to choose a suitable basis which allows high accuracy for a small number of terms $n_{B}<N_{B}$; for a prior characterized system such a set of basis functions, as well as the corresponding expansion coefficients, may be obtained via computation of the Singular Value Decomposition (SVD) of B [4].

\section{RESULTS}

In order to illustrate the trade-off between computational efficiency and calculation accuracy over a wide frequency band, snapshot observations based on using the Low Band Array (LBA) of a single LOFAR [5] station as an interferometric imaging array were simulated. The array operates from $10-90 \mathrm{MHz}$ and observations were simulated at $40 \mathrm{MHz}$, $50 \mathrm{MHz}, 60 \mathrm{MHz}$, and $70 \mathrm{MHz}$. Radiation patterns of the antennas in the array were simulated at these frequencies in FEKO [6]. A model sky was produced by randomly placing ten point sources on a $16 \times 16$ image grid, and then used as input to calculate the reference visibilities measured by the array using (3). Model visibilities were then calculated using various numbers of terms in (6) (i.e. truncating the sum after $n_{B}<N_{B}$ terms) with which to approximate the directiondependent effects in $\mathbf{B}$, and the error between the measured and modeled visibilities was determined in each case as,

$$
\epsilon=\left\|\mathbf{v}_{\text {meas }}-\mathbf{v}_{\text {model }}\right\|_{2} /\left\|\mathbf{v}_{\text {meas }}\right\|_{2} .
$$

Fig. 1 shows the error in the forward calculation as a function of the number of terms $n_{B}$, and compares the results obtained at different frequencies. Due to mutual coupling effects, that were fully accounted for in the simulation, the radiation patterns of the antennas exhibit significant interelement variability at higher frequencies [7]. As a result a larger number of terms is required to perform the forward

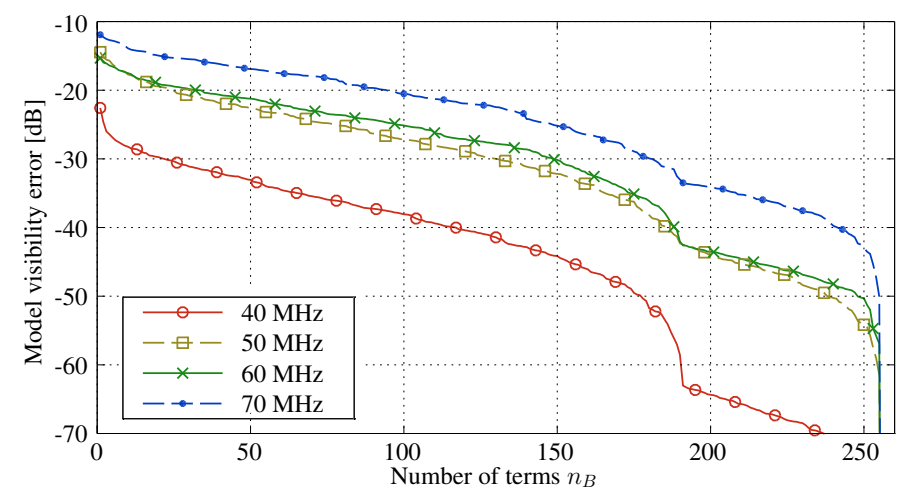

Fig. 1. Error in model visibilities as a function of the number of terms $n_{B}$ retained in the A-stacking model. For the LOFAR LBA more terms are required to model direction-dependent effects to a certain accuracy level at higher frequencies than at lower frequencies.

calculation with a given level of accuracy for the LOFAR LBA at high frequencies as compared to at lower frequencies.

\section{CONCLUSION}

The A-stacking framework presents an efficient method for modeling the response of an interferometric imaging array, while accounting for direction-dependent effects that are also baseline-dependent. It also provides a useful trade-off between computational complexity and calculation accuracy; here this trade-off was demonstrated for a wideband imaging array, where the same level of accuracy may require different numbers of terms at various frequencies.

\section{ACKNOWLEDGEMENT}

This work is supported by SKA South Africa, the South African Research Chairs Initiative of the Department of Science and Technology, the National Research Foundation, and the Swedish Vinnova and VR grants. This publication is supported by Samenwerkingsverband Noord Nederland (SNN), SKA-TSM project, and the European Community FP7 program, MIDPREP, Grant Agreement PIRSES-GA-2013612599.

\section{REFERENCES}

[1] A. R. Thompson, J. M. Moran, and G. Swenson, Interferometry and Synthesis in Radio Astronomy, 2nd ed. Wiley, 2004.

[2] J. I. Jackson, C. H. Meyer, D. G. Nishimura, and A. Macovski, "Selection of a convolution function for Fourier inversion using gridding," IEEE Trans. Medical Imaging, vol. 10, no. 3, pp. 473-478, 1991.

[3] O. M. Smirnov, "Revisiting the radio interferometer measurement equation II: Calibration and direction-dependent effects," $A \& A$, vol. 527, p. A107, 2011.

[4] A. Young, S. J. Wijnholds, T. D. Carozzi, R. Maaskant, M. V. Ivashina, and D. B. Davidson, "Efficient Correction for Both Baseline-Dependent and Direction-Dependent Effects in Interferometric Imaging: An AStacking Framework," $A \& A, 2014$, under review.

[5] M. De Vos, A. Gunst, and R. Nijboer, "The LOFAR Telescope: System Architecture and Signal Processing," vol. 97, no. 8, pp. 1431-1437, Aug. 2009.

[6] FEKO Suite 7.0. [Online]. Available: https://www.feko.info/

[7] A. Young, T. Carozzi, R. Maaskant, M. V. Ivashina, and D. B. Davidson, "Toward a practical demonstration of physics-based beam calibration models - CBFPs and the LOFAR-LBA antenna array radio telescope," in International Conference on Electromagnetics in Advanced Applications (ICEAA), 2014, pp. 462-465. 